\title{
The Deformation of Expanded Clay Syntactic Foams during Compression Characterized by Acoustic Emission
}

\author{
Csilla Kádár ${ }^{1,2 *}$, Kristián Máthis ${ }^{3}$, František Chmelík ${ }^{3}$, Michal Knapek ${ }^{3,4}$, Imre N. Orbulov ${ }^{1,2}$ \\ ${ }^{1}$ Department of Materials Science and Engineering, Budapest University of Technology and Economics, \\ Müegyetem rakpart 3., H-1111 Budapest, Hungary \\ ${ }^{2}$ MTA-BME Lendület Composite Metal Foams Research Group, Mủegyetem rakpart 3., \\ H-1111, Budapest, Hungary \\ ${ }^{3}$ Department of Physics of Materials, Charles University, Ke Karlovu 5, CZ12116 Prague 2, \\ Czech Republic \\ ${ }^{4}$ Nuclear Physics Institute, Czech Academy of Sciences, Řež 130, 25068 Řež, Czech Republic
}

\begin{abstract}
The deformation and failure mechanisms in syntactic foams with different metal matrices were investigated in this study. The syntactic foams were produced by the infiltration method using lightweight expanded clay particles (LECA) as a space holder and commercially pure $\mathrm{Al}$ or eutectic $\mathrm{Al}-12 \% \mathrm{Si}$ alloy for the matrices. The samples were compressed at room temperature; simultaneously, the acoustic emission (AE) response and the surface deformation were monitored and collated with the deformation curves. In the case of the $\mathrm{Al}$ foam, ductile behavior with long plateau stress was observed. During this plateau regime, multiple active shear bands were detected. In contrast, the AlSi12 foam exhibited more brittle deformation behavior. At the end of the quasi-linear stage, the localization of the strain started within one large shear band, accompanied by a significant stress drop. The AE analysis revealed that the deformation was mostly governed by the fracture of LECA particles and the plastic deformation of the matrix material for both types of foams. After strain localization, in the case of the AlSi12 foam, the fracture of the matrix became significant, causing the deterioration of the sample. As for the Al foam, besides the fracture of the LECA particles, the plastic deformation of the matrix played an important role in preventing the foam from falling apart.
\end{abstract}

Keywords: Metal matrix syntactic foams, Deformation, Acoustic emission

\section{Introduction}

In recent years, research on the mechanical properties of metal matrix foams and metal matrix syntactic foams (MMSF) have received extensive attention since these materials are lightweight structures with high strength-to-weight and stiffness-to-weight ratios, with good damping and energy absorption properties [1-3].

There are different strategies for improving the strength and the energy absorption capacity of a foam. One option is to increase the strength of the matrix material. This can be achieved by a) heat treatment, b) adding alloying elements to generate intermetallic phases or c) using micro- or nano-sized reinforcing particles such as $\mathrm{SiC}$ or alumina particles, or carbon nanotubes [2]. However, there is another way to enhance the mechanical properties of a foam by incorporating low density, stiff and porous particulates (filler material) into the matrix. The materials produced in this way are referred to as syntactic foams [4].

The most frequently used additions for manufacturing syntactic foams are the hollow spheres. Mostly, ceramic hollow spheres are used [5-7], however, recently, MMSF with steel hollow spheres have also been produced [8]. Although hollow spheres enhance the plateau stress of the foam considerably, due to the high cost of producing new low-cost

* Corresponding author. E-mail addresses: kadar@metal.elte.hu (Cs. Kádár), 
filler materials are needed. Up to now, syntactic foams with expanded glass [9], pumice [10], expanded perlite [1], and fly ash [4] fillings have been investigated.

Recently, lightweight expanded clay (LECA) as a filler material has also been designed as it features high porosity, low density, and good thermal/acoustic insulation properties [11,12]. So far only the effect of the LECA particle diameter has been investigated [12]. Puga and co-workers have found that with increasing LECA particle diameter both the yield strength and the densification strain increase. They found a change in deformation mechanism: for foams containing large LECA particles the deformation is less uniform [12].

In this paper, the effect of the matrix material on the failure mechanisms and compressive strength of MMSF containing LECA particles was studied. The changes in deformation mechanisms were also investigated by the acoustic emission (AE) and digital image correlation (DIC) techniques.

\section{Experimental}

The matrix materials of the investigated syntactic foams are commercially pure aluminum (Al) and an AlSi12 alloy. The syntactic foam was produced by low-pressure infiltration, as follows. In a closed profile crucible of a cross-section of $50 \mathrm{~mm} \times 50 \mathrm{~mm}$ first sieved LECA particles having a diameter of 6-7 mm, then a stainless steel grid (to hold them in position during infiltration) and a block of metal (AlSi12/Al) were placed. Next, the crucible (closed by welding) was heated up and held at $750{ }^{\circ} \mathrm{C}$ for $\mathrm{Al}$ and $650{ }^{\circ} \mathrm{C}$ for $\mathrm{AlSi} 12$ for $30 \mathrm{~min}$. To complete the infiltration, inert gas (He) was applied for $120 \mathrm{~s}$ at 1 bar pressure. Subsequently, the block was quenched into water of ambient temperature. Finally, $4 \mathrm{~mm}$ of the side parts and the bottom of the obtained foam block were mechanically removed to ensure a homogeneous foam structure.

An MTS810 type servo-hydraulic universal testing machine (MTS Systems Corporation, Eden Prairie, MN, USA) was used to carry out the uniaxial compression tests with cyclic loading at room temperature. Three cube-shaped foam specimens of each type with a side length of $30 \mathrm{~mm}$ and a density of $1.55 \pm 0.05 \mathrm{~g} / \mathrm{cm}^{3}$ were compressed at a crosshead speed of $0.01 \mathrm{~mm} / \mathrm{s}$. Teflon strips were applied between the foams and machine grips to reduce interface friction. The deformation of the surface was recorded by a video camera. The digital image correlation (DIC) technique was applied to determine the strain localization during deformation by using the ncorr script implemented in Matlab [13].

Simultaneously with the compression test, the AE activity was monitored by a Vallen AMSY-6 System in transient recording mode with a sampling rate of $1 \mathrm{MHz}$. The AE signal recorded using the Physical Acoustic Corporation WS $\alpha$ sensor was pre-amplified with a gain of $40 \mathrm{~dB}$. The sensor was mounted on the surface of the specimen using vacuum grease and an elastic band.

The structures of the syntactic foams and the LECA particles were investigated by a Hitachi TM4000 Tabletop scanning electron microscope.

\section{Results and discussion}

Representative engineering stress-strain curves with different matrix materials are shown in Fig. 1. In the case of unalloyed Al-LECA foam, ductile behavior can be observed (Fig. 1a), i.e. after the quasi-linear region the stress reaches a plateau, after which there is practically no stress drop. In the plateau region, the deformation of the cells in the shear band(s) takes place. Finally, in the densification stage, the matrix material fills the remaining pores produced by the crumbling of the LECA particles. Contrary to the Al foam, the AlSi12-LECA foam does not exhibit distinct plateau stress, but rather peak stress followed by a stress drop occurs. The peak stress of the AlSi12 foam is roughly twice as large as the peak stress of the $\mathrm{Al}$ foam. It is worth noting that even though the samples are quite small compared to the size of the LECA-particles, the stress-strain response is reproducible for Al-LECA foams (see Fig. 1a), while for AlSi12-LECA the shape of the deformation curve depends on structure of the foam specimen. Nevertheless, the variation of the peak stress was less than $10 \%$. 

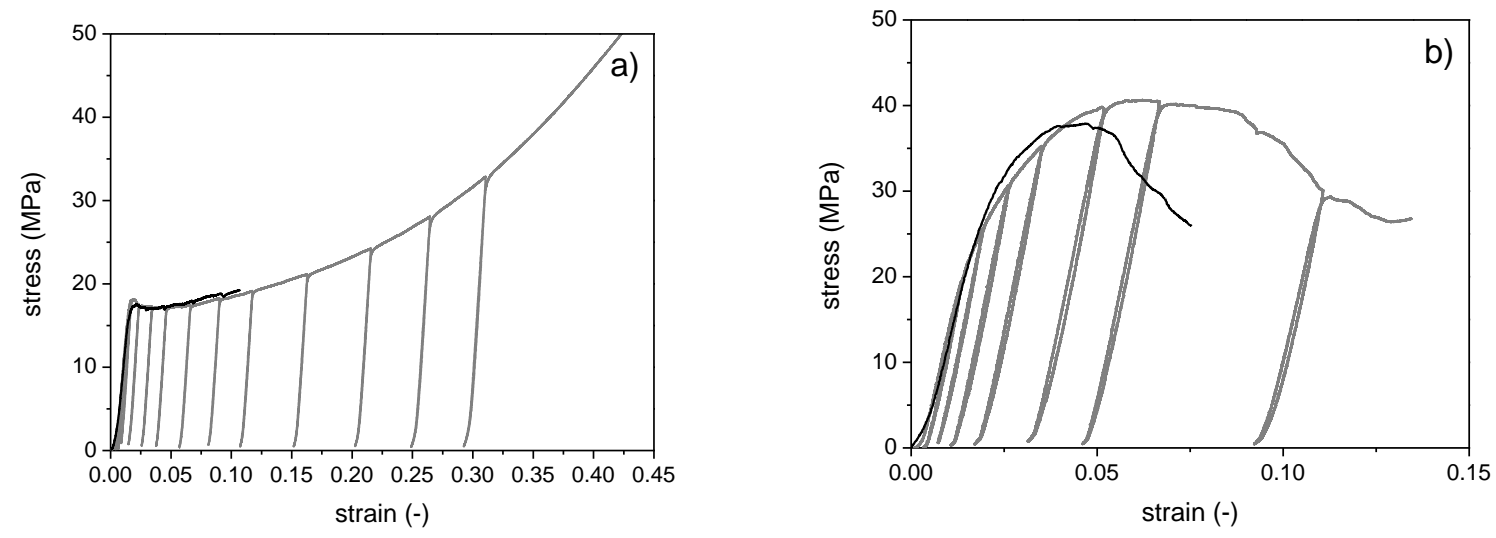

Fig. 1. The stress-strain curves (black line) for metal matrix syntactic (MMS) foams containing expanded clay with (a) Al matrix; (b) AlSi12 matrix.

The $\mathrm{AE}$ response during compression is dependent on the matrix material. The $\mathrm{AE}$ response shows a maximum around the peak stress in the case of Al-LECA foam, while for the AlSi-LECA foam the maximum AE activity can be measured before the peak-stress is reached. In both cases, the AE peak is followed by a considerable drop in the AE activity, especially for the AlSi12-LECA foam (Fig. 2). This drop is due to the localization of deformation. Due to strain localization, the number of active AE sources decreases and, correspondingly, the AE activity reduces.

The adaptive sequential k-means (ASK) clustering analysis was performed on the AE data to determine the dominant deformation mechanism as a function of strain. The $\mathrm{AE}$ data are sectioned to time windows (in this evaluation $1 \mathrm{~ms}$ time window was used) and the power spectral density function statistical parameters (such as mean frequency, peak amplitude, energy, kurtosis, etc.) of each time window are determined and grouped into clusters according to the algorithm of Pomponi and Vinogradov. The detailed description of the method can be found in [14]. For the identification of the AE sources, complementary methods like digital image correlation (DIC) and scanning electron microscopy (SEM) were used. It is important to emphasize that if at a given strain multiple different AE sources are active, the ASK method is only capable of determining the dominant one.

At the beginning of the test, the cluster of background noise should be identified. To that end, the AE measurement is started prior to the compression. During the test, the ASK algorithm identified four clusters for both, $\mathrm{Al}$ and AlSi12 foams. Fig. 2 shows the time evolution of the cumulative number of elements for the different AE clusters. The following source mechanisms were assigned to the clusters:
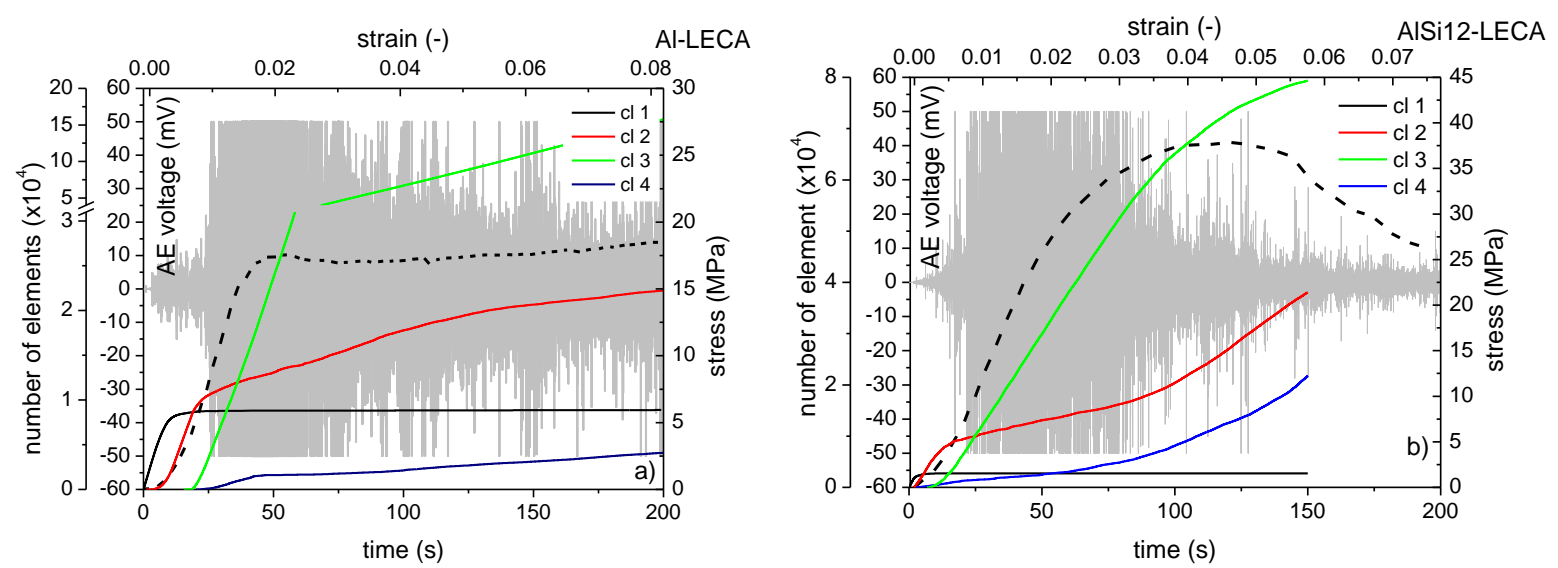
Fig. 2. Representative compressive stress-strain curves (dashed line), acoustic emission (AE) signals (grey line) and the time evolution of the cumulative number of elements in different AE clusters of (a) Al foam; (b) AlSi12 foam. Black line - noise; red line — plastic deformation of matrix; green line - fracture of LECA particles; blue linefracture of the matrix.

Cluster 1: Noise (color code in figures: black)

The cluster appearing first is always the noise cluster, since the AE test starts prior to the deformation and, therefore, the noise cluster is not directly related to the deformation processes. This cluster contains low energy AE signals with low amplitude and no sharp peaks.

Cluster 2: Plastic deformation of the matrix (color code in figures: red)

In both cases, the second cluster appears at very low strain. Similarly to the analysis in our previous work [15] (e.g. the drop shape of the cluster (Fig. 3), the shape of the waveform), plastic deformation can be assigned to Cluster 2.

Cluster 3: fracture of LECA particles (color code in figures: green)

The signals belonging to this cluster exhibit a negligible rise time, furthermore, the energy range of these signals is wide, suggesting that the origin of AE is the fracture of LECA cell-walls of different thicknesses. The cracking of the LECA cell walls is visible at low stress in the SEM pictures (Fig. 4a).

Cluster 4: fracture of the matrix (color code in figures: blue)

As for the AE signals in Cluster 3, the signals in Cluster 4 have wide frequency and energy range and a negligible rise time. The video recording shows that in the case of the AlSi12 foam the role of matrix fracture becomes significant after strain localization. Fig. $2 b$ shows that the cumulative number of elements in Cluster 4 suddenly increases when the $\mathrm{AE}$ activity decreases. It is indeed expected that due to strain localization the fracture of the matrix becomes more dominant (Fig. 4c). In the case of the $\mathrm{Al}$ foam, the fracture of thin cell walls between LECA particles is visible after strain localization.
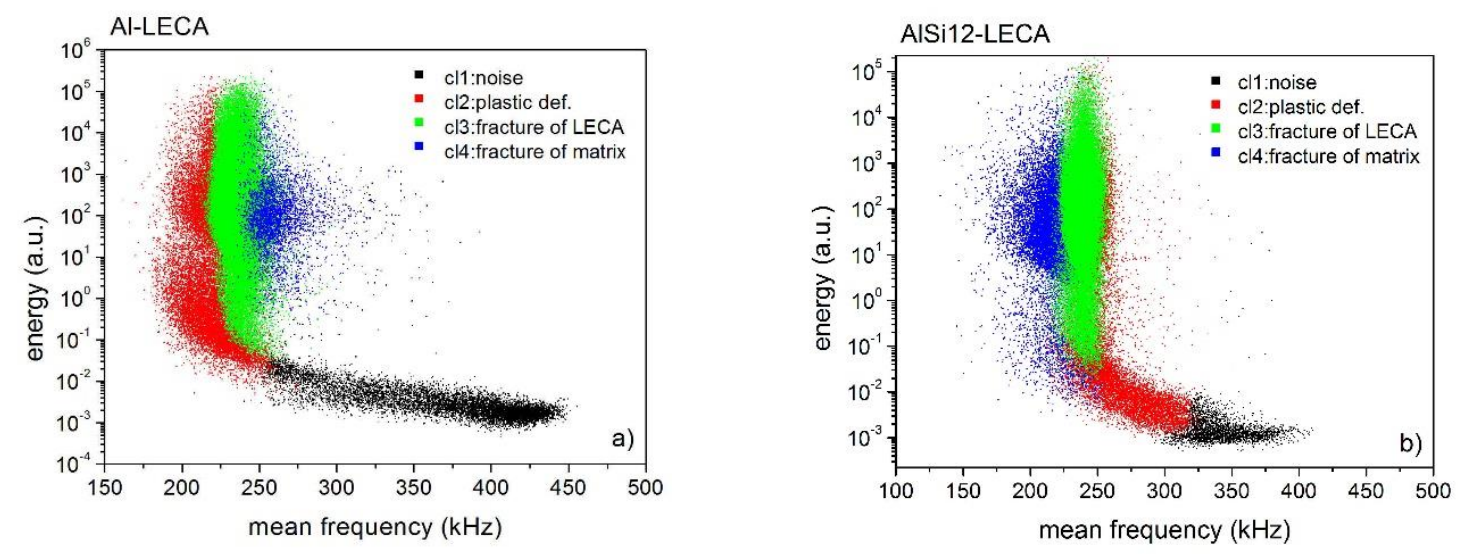

Fig. 3 The 2D projection of the clusters to the energy-mean frequency space for (a) Al foam; (b) AlSi12 foam. 

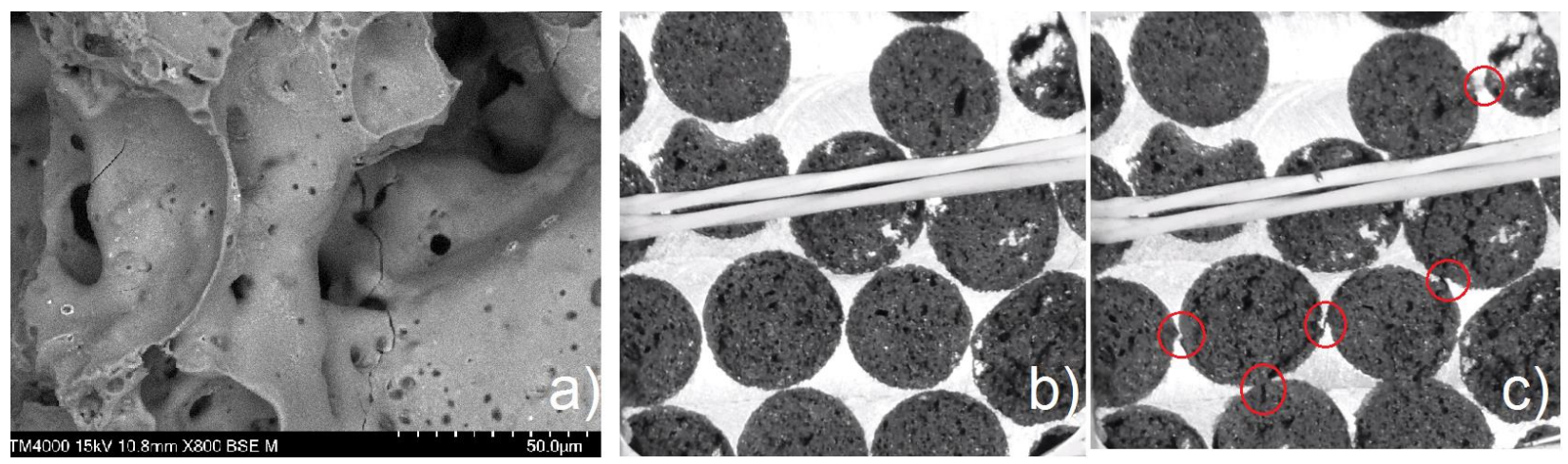

Fig. 4. The deformation of the reinforcement and the foam. (a) Cracks in the LECA particle in the deformed foam, (b) undeformed AlSi12 LECA foam and (c) compressed AlSi12 LECA foam (The places where plastic deformation or fracture of the matrix took place are marked by red circles).

The results of the ASK analysis show that the Al LECA foam first deforms by plastic deformation of the matrix material. Due to the low strength of the LECA particles, the cracking of the LECA spheres starts at low applied stress ( $\sim 5 \mathrm{MPa}$ ). The localization of the strain starts at the end of the quasi-linear stage (Fig. 5), and the formation of deformation bands can be subsequently observed. Contrary to conventional foams, the deformation of the Al LECA foam is not restricted to only one band (Fig. 5). As a result, the AE activity decreases due to strain localization, but the drop in AE activity is only moderate since the deformation still takes place in a relatively large volume. After the appearance of deformation bands, the rate of growth in the number of elements in Cluster 2 decreases, while the number of elements in Cluster 3 increases linearly as a function of strain. It implies that the dominant deformation mechanism is the fracture of LECA particles throughout the deformation process.

For small strains the AlSi12 foam deforms similarly to the $\mathrm{Al}$ foam, i.e. plastic deformation of the matrix takes place almost as soon as the compression starts, followed by the beginning of fracture of the cell walls of LECA particles at about $2 \mathrm{MPa}$ applied stress. The lower onset of the LECA particles' crack is due to the higher back stress of the AlSi12 foam compared to the $\mathrm{Al}$ foam. (The back stress was calculated from the unloading and reloading according to [16]). After the appearance of the shear band, the rate of growth in the number of elements in Cluster 2 and 4 increases, while for Cluster 3 decreases. This suggests that in the AlSi12 LECA foam after strain localization the fracture of the matrix becomes important (Fig. 4c).
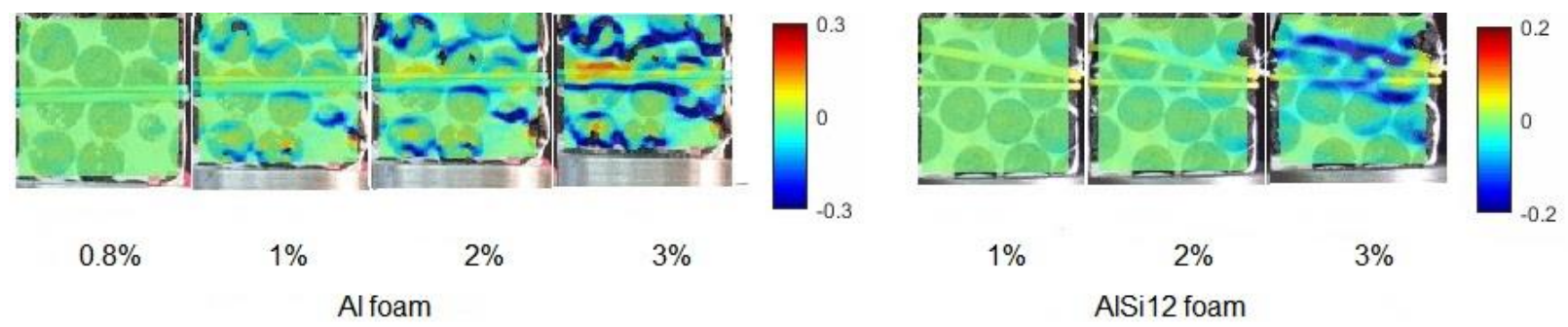

Fig. 5. Surface $\varepsilon_{y y}$ strain maps at different macroscopic strains for LECA foams with different matrixes: Al (left) and AlSi12 (right).

It is worth noting that according to Fig. 2, the fracture of the cell walls of the LECA particles is the dominant deformation mechanism almost for the entire deformation test. This means that weaker AE signals from other sources are overlapped. This is most likely the reason why the number of elements in the cluster related to plastic deformation in the Al-LECA foam is lower than for the Al foam with ceramic hollow spheres [15].

\section{Conclusion}

The effect of the matrix material on deformation and failure mechanisms was investigated in two types of syntactic foams consisting of (i) unalloyed Al matrix or (ii) alloyed Al: Al-12 wt \% Si matrix, both reinforced by lightweight expanded clay particles. The differences in deformation mechanism up to $\sim 6 \%$ strain were revealed by the help of 
the ASK analysis of AE data supplemented by imaging techniques. We found that regardless of the matrix material, the identified deformation mechanisms were rather similar, and there was no strain localization in the quasi-linear stage. This resulted in reproducible peak strength with a variation of less than $10 \%$, even for samples with small sample size to LECA particle size ratio. In the case of $\mathrm{Al}$ foam, the strain is localized in large volume in multiple deformation bands, which results in reproducible deformation curves even for small samples. After strain localization, the fracture of the matrix material became significant for the AlSi12 foam. This explains the difference in the shape of the stress-strain curves for the AlSi12 foam and the early deterioration of the sample.

\section{Acknowledgments}

The authors are grateful for the financial support of the Czech Science Foundation under the contract 15-10821S. This paper was supported by the János Bolyai Research Scholarship of the Hungarian Academy of Sciences (Cs. Kádár). M. Knapek gratefully acknowledges partial financial support from the OP RDE, MEYS, grant No. CZ.02.1.01/0.0/0.0/16_013/0001794.

\section{References}

[1] Taherishargh M, Belova IV, Murch GE, Fiedler T (2014) Low-density expanded perlite-aluminium syntactic foam. Mat Sci Eng A 604:127-134. doi:10.1016/j.msea.2014.03.003.

[2] Duarte I, Ferreira JMF (2016) Composite and Nanocomposite Metal Foams. Materials 9:79. doi:10.3390/ma9020079.

[3] Gupta N, Rohatgi PK (eds.) (2015) Metal matrix syntactic foams: processing, microstructure, properties and applications. DEStech Publications, Lancaster

[4] Rohatgi PK, Gupta N, Schultz BF, Luong DD (2011) The synthesis, compressive properties, and applications of metal matrix syntactic foams. JOM 63:36-42. doi:10.1007/s11837-011-0026-1.

[5] Balch DK, O’Dwyer JG, Davis GR, Cady CM, Gray GT, Dunand DC (2005) Plasticity and damage in aluminum syntactic foams deformed under dynamic and quasi-static conditions. Mat Sci Eng A, 391:408-417.

doi:10.1016/j.msea.2004.09.012.

[6] Santa Maria JA, Schultz BF, Ferguson JB, Rohatgi PK (2013) Al-Al2O3 syntactic foams - Part I: Effect of matrix strength and hollow sphere size on the quasi-static properties of Al-A206/A12O3 syntactic foams. Mat Sci Eng A 582:415-422. doi:10.1016/j.msea.2013.05.081.

[7] Orbulov IN, Ginsztler J (2012) Compressive characteristics of metal matrix syntactic foams. Compos Part AAppl S 43:553-561. doi:10.1016/j.compositesa.2012.01.008.

[8] Szlancsik A, Katona B, Bobor K, Májlinger K, Orbulov IN (2015) Compressive behaviour of aluminium matrix syntactic foams reinforced by iron hollow spheres. Mater Design 83:230-237. doi:10.1016/j.matdes.2015.06.011

[9] Al-Sahlani K, Broxtermann S, Lell D, Fiedler T (2018) Effects of Particle Size on the Microstructure and Mechanical Properties of Expanded Glass-Metal Syntactic Foams. Mat Sci Eng A 728:80-87.

doi:10.1016/j.msea.2018.04.103.

[10] Taherishargh M, Belova IV, Murch GE, Fiedler T (2015) Pumice/aluminium syntactic foam. Mat Sci Eng A 635:102-108. doi:10.1016/j.msea.2015.03.061.

[11] Bazzaz Bonabi S, Kahani Khabushan J, Kahani R, Honarbakhsh RA (2014) Fabrication of metallic composite foam using ceramic porous spheres "Light Expanded Clay Aggregate" via casting process. Mater Design 64:310315. doi:10.1016/j.matdes.2014.07.061. 
[12] Puga H, Carneiro VH, Jesus C, Pereira J, Lopes V (2018) Influence of particle diameter in mechanical performance of $\mathrm{Al}$ expanded clay syntactic foams. Compos Struct 184:698-703.

doi:10.1016/j.compstruct.2017.10.040.

[13] Blaber J, Adair B, Antoniou A (2015) Ncorr: Open-source 2D digital image correlation Matlab software. Exp Mech 55:1105-1122. doi:10.1007/s11340-015-0009-1.

[14] Pomponi E, Vinogradov A (2013) A real-time approach to acoustic emission clustering. Mech. Syst Signal Process 40:791-804. doi:10.1016/j.ymssp.2013.03.017.

[15] Kádár C, Máthis K, Knapek M, Chmelík F (2017) The Effect of Matrix Composition on the Deformation and Failure Mechanisms in Metal Matrix Syntactic Foams during Compression. Materials 10:196. doi:10.3390/ma10020196.

[16] Yang M, Pan Y, Yuan F, Zhu Y, Wu X (2016) Back stress strengthening and strain hardening in gradient structure. Mater Res Lett 4:145-151. doi:10.1080/21663831.2016.1153004. 